\title{
PERSPECTIVES
}

INNOVATION

\section{Current status and future prospects of needle-free liquid jet injectors}

\section{Samir Mitragotri}

Abstract | Needle-free liquid jet injectors have been used for more than 50 years for parenteral delivery of vaccines and drugs. Although excellent bioavailability has been reported for a number of drugs, occasional pain and bruising have limited wide acceptance of jet injectors. This article reviews jet injectors with respect to their current clinical applications, emerging applications, mechanistic understanding and future prospects.

Most vaccines and protein therapeutics are currently delivered using needles and syringes. Despite their common use, needlebased methods have several limitations, such as needle phobia ${ }^{1}$, accidental needle-stick injury ${ }^{2}$, and re-use of needles and syringes in developing countries ${ }^{3}$ that leads to a large number of HIV and hepatitis B virus (HBV) cases $^{4}$. Several methods are under investigation for needle-free drug and vaccine delivery ${ }^{5-9}$. Among these, needle-free liquid jet injections stand out as the method with the longest history in delivering macromolecules in humans. So far, liquid jet injectors have been used to deliver several macromolecules, including vaccines, insulin and growth hormones in a large number of patients ${ }^{6,10-12}$, and erythropoietin ${ }^{13}$ and interferon in a few cases $^{14}$. Jet injectors have also been used to deliver small molecules such as lidocaine $e^{15,16}$, midazolam ${ }^{17}$ and ketamine ${ }^{18}$. In this article the historical development and current and emerging clinical applications of needle-free liquid jet injectors are reviewed. Mechanistic considerations related to effective jet injection are discussed and the future prospects of liquid jet injectors are considered. An emphasis is placed on mechanistic understanding because this topic has not been previously reviewed in the literature.

\section{Jet injection}

The origin of jet injections dates back to the late 1800s when a technique known as aquapuncture was reported in the medical literature ${ }^{19}$. This device was used to deliver jets of water and other liquids for applications other than drug delivery, such as treatment of uncontrolled neuralgia. The modern history of jet injectors for drug delivery began in the 1930s. Since then, several types of jet injector have been developed and used in clinical applications (BOX 1). Current liquid jet injectors use a high-velocity jet (typically $>100 \mathrm{~m} \mathrm{~s}^{-1}$ ) to deliver molecules across the skin into the subcutaneous or intramuscular region. The typical diameter of the jet used in commercial injectors is $76-360 \mu \mathrm{m}$, which is smaller than the outer diameter of a standard hypodermic needle ( $810 \mu \mathrm{m}$ for a $21 \mathrm{G}$ needle).

Jet injectors can be broadly classified into multi-use nozzle jet injectors (MUNJIs) and disposable cartridge jet injectors (DCJIs), depending on the number of injections carried out with a single device. MUNJIs provide repeated injections from a single reservoir and were once popular for mass immunization. Today, MUNJIs are not used in human applications; however, they continue to be used for veterinary applications. MUNJIs have had a significant impact on the field of jet injections and are therefore discussed in this article. DCJIs deliver a single injection from a drug reservoir and are the most commonly used form of jet injectors today. Some DCJIs are only partly disposable (a disposable liquid reservoir in conjunction with a non-disposable actuation mechanism) whereas others are fully disposable (BOX 1).

\section{Clinical applications}

A number of different drugs and vaccines have been delivered in humans using jet injections. In this article, emphasis is placed on three examples - vaccines, insulin and growth hormones - because they are all macromolecules that otherwise require needles for their delivery, and they are among the most important clinical applications of jet injections. Other drugs that have been delivered in humans by jet injectors will be mentioned only briefly.

Immunization. Liquid jet injections for immunization were first carried out using MUNJIs, which allowed repeated injections of vaccine from the same nozzle and reservoir at a rate of up to 1,000 immunizations per hour. They were successfully adopted for immunizing humans using vaccines against a large number of diseases, including measles, smallpox, cholera, HBV, influenza and polio ${ }^{20}$. MUNJIs have had an especially significant role in the efforts to eradicate smallpox ${ }^{21}$. The effectiveness of MUNJIs for immunization, as judged by seroconversion, is at least as good as that of needle-based immunizations. On certain occasions they have resulted in higher seroconversion rates, possibly due to wider distribution of the vaccine in the body and enhanced local reactions ${ }^{22}$. The primary drawback of MUNJIs has been the potential for subjectto-subject contamination, which was discovered through the spread of $\mathrm{HBV}^{23}$. Splashes of small amounts of liquid, contaminated with blood or interstitial fluid, on the nozzle of the jet injector were blamed for the HBV spread. Recent studies with high-speed photography of jet injection into human skin in vitro have provided details of liquid splash (FIG. 1), which, in its simplest interpretation, represents liquid bouncing off the skin and back towards the nozzle. In light of the increased risk of contamination, the World Health Organization recommends that MUNJIs should not be used for immunization until all safety issues have been resolved. By contrast, the Centers for Disease Control and Prevention recommend that MUNJIs should be used for mass immunization only when the gains from rapid immunization outweigh the risks of blood-borne diseases, 


\section{Box 1 Historical development and current status of jet injector designs}

The earliest descriptions of jet injections date back to the nineteenth century, whereas the modern era of liquid jet injectors began in the early 1930s. Spring- or gas-powered devices were developed for single or multiple injection applications. Spring-powered devices have the advantages of compactness, low cost and high durability. Their disadvantages include a limited range of force and reduced versatility. Gas-powered devices offer the advantage of sustained force generation, greater flexibility and the capacity to deliver large volumes. Their disadvantages include complexity and reliance on an exhaustible energy source ${ }^{76}$. Jet injectors were first popularized in the form of multi-use nozzle jet injectors (MUNJIs), which were introduced in the US military between 1947 and 1965 because they offer a high rate of vaccination. MUNJIs delivered vaccines from a single-dose vial at a rate of up to 1,000 per hour. The use of MUNJIs decreased drastically after evidence of contamination originating from blood and splashed liquid was uncovered. At around the same time that MUNJIs were popularized, single-dose devices were also developed for insulin delivery. A device called the Hypospray was introduced onto the market in 1961 for insulin delivery ${ }^{10}$. Although Hypospray was discontinued in 1971, other devices were introduced for single-dose administration. These injectors were non-disposable and required thorough cleaning between injections. Disposable cartridge jet injectors (DCJls) were introduced in the late 1990 s to facilitate use and cleanliness. Clear plastic nozzles that hold the drug not only facilitated cleanliness by clearly separating the drug from the actuation device but also helped in drug loading. Today DCJls remain the primary type of jet injectors in use. The latest form of DCJls includes single-use, pre-filled, completely disposable injectors, which further facilitates their use and reduces risk of contamination. such as during pandemic influenza or bioterrorism attacks ${ }^{24}$. Detailed protocols for cleaning the injector have been proposed to minimize the risk of contamination ${ }^{25}$; however, their effectiveness in clinical situations remains to be determined. To minimize the risk of contamination, protective, disposable devices that cover the surface of the injector have been developed, and studies carried out with these devices showed no risk of contamination in a population of 22,714 participants ${ }^{26}$. The injection volumes used in this study, however, were small $(0.1-0.2 \mathrm{ml})$ and the utility of the protection devices for larger volumes remains to be confirmed.

Although MUNJIs are no longer used for routine immunizations, DCJIs are used for both childhood and adult vaccination at the physician's discretion. However, the acceptability of DCJIs for immunization has been mixed. Some studies have reported that DCJIs induce higher levels of pain compared with needles ${ }^{27}$, whereas others have found no difference between the two methods ${ }^{28}$. Jet injectors have been associated with more frequent local-site reactions, such as soreness, redness and swelling of the injection site, than needle-based injections ${ }^{22,29}$. The number of immunizations carried out today with DCJIs is far less than the number of needle-based immunizations. High cost and low awareness among healthcare providers and patients also add to reduced enthusiasm for DCJI-based immunization.

Insulin. Non-compliance with needles among diabetic patients has been one of the primary motivations for using jet injectors (specifically DCJIs) for insulin delivery. In addition, jet injections are thought to be easier to use for patients with impaired motor skills. Jet injectors hold special significance in the field of diabetes management. They represent the first commercial needle-free method for insulin delivery and the only such method until the recent FDA approval of inhaled insulin. Insulin delivery by jet injections is as effective as needles, as judged by control of blood glucose level ${ }^{30-32}$. A more rapid increase in plasma insulin levels is typically observed with jet injections s $^{33,34}$, possibly due to better dispersion of insulin at the site of injection than with needles ${ }^{30}$. A large number of insulin doses have been administered by jet injection to diabetic patients, although the exact number is difficult to track. It is clear, however, that the number of patients using jet injectors is far less than those using needles and syringes.

The major concern with jet-injected insulin has been variable adverse reactions. Some studies have reported no local reactions after jet injections of insulin ${ }^{35}$, whereas others have reported significant reaction, including immediate pain, delayed pain, bleeding and haematomas ${ }^{36}$. Bruising and bleeding have been reported in about $30 \%$ of insulin injections in some studies ${ }^{10}$. Several studies have concluded that jet injections are no less painful than needles ${ }^{37}$, leading to mixed acceptance among patients $s^{35,38}$. Studies have also reported difficulties with the proper use of jet injectors among young diabetic children ${ }^{39}$. Improper functioning of the device can lead to 'wet' injections in which the jet is not able to properly penetrate the skin, or can lead to bruising and pain if the jet penetrates excessively deeply into the body.

Growth hormones. Jet injectors have had a significant impact on the delivery of growth hormone, more so than on any other therapeutic drug. The target population for growth hormone delivery is juvenile, thereby increasing the appeal of needle-free liquid jet injectors. A higher and earlier maximum blood concentration was found after jet injection of recombinant human growth hormone compared with needle injections ${ }^{40}$. Jet-injected and needle-injected recombinant growth hormones were found to be bioequivalent with respect to the amount

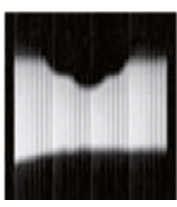

0

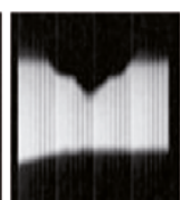

$40 \mu \mathrm{s}$

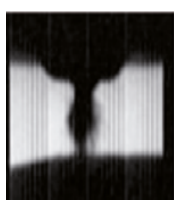

$160 \mu \mathrm{s}$

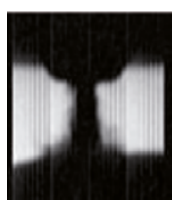

$280 \mu \mathrm{s}$

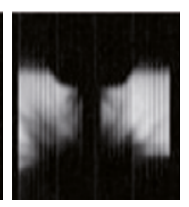

$400 \mu \mathrm{s}$

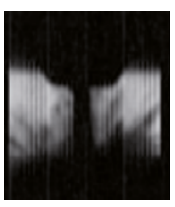

$520 \mu \mathrm{s}$

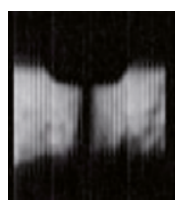

$720 \mu \mathrm{s}$

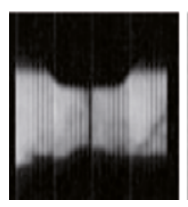

$1 \mathrm{~ms}$

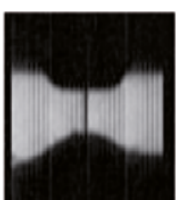

$2 \mathrm{~ms}$

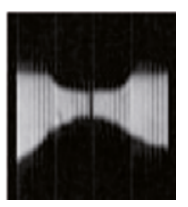

$5 \mathrm{~ms}$
Figure 1 | Snapshots from high-speed video of jet injection of water into human skin in vitro. The black region at the top of each image shows the outline of a jet injector with the protrusion in the centre showing the position of the nozzle. The black region at the bottom shows the outline of human skin. The gap between the nozzle and the skin is a few millimetres. At time $t=0$, the injector is activated and a jet begins to emerge towards the skin. The jet makes first contact with the skin at
$160 \mu \mathrm{s}$. The initial impact of the jet on the skin produces some splash. The typical volume of the liquid splashed in the image at $400 \mu \mathrm{s}$ is around 100 nl. The jet subsequently assumes a tight cylindrical form and deposits the liquid into the skin. Deposition of liquid causes the skin to bulge, which is evident at 2-5 ms. More than $90 \%$ of the ejected fluid is deposited in the skin under these conditions. The occurrence and extent of the bulge depends on injection volume. Images courtesy of J. Baxter. 
absorbed ${ }^{41}$ but not the rate of absorption. Acceptability of jet injections of growth hormone has been mixed, like insulin: some studies have reported good tolerance of jet injections ${ }^{40}$, whereas others have reported more bleeding, pain, soreness and bruising than with needles ${ }^{11,12}$. A study conducted on juveniles found jet injections to be less painful (including both frequency and intensity of pain) than a $28 \mathrm{G}$ needle; however, the prevalence of visible bruises was higher with jet injections than with needle injections ${ }^{42}$.

Other drugs. Several other small-molecule and macromolecular drugs have been delivered using jet injectors. The broad use of jet injectors is due to the fact that they can work with formulations developed for needle-based injections, thereby minimizing the barrier to new applications. Anaesthetics such as lidocaine have been commonly delivered using jet injections ${ }^{43-45}$ for various applications, including dental procedures, dermal surgical procedures and peripheral intravenous cannula insertion. Jet-delivered anaesthetic is generally found to be equivalent to needle-based injections in terms of effectiveness and better than needle-based injections in terms of acceptance. Sedatives such as midazolam have also been delivered using jet injections ${ }^{46}$ and have produced pharmacological effects equivalent to needle-based injections. Jet injectors have also been used for local delivery of steroids directly into the lesion for treatment of scars $^{47}$. Intralesional bleomycin has also been safely and effectively used for treating keloids and hypertrophic scars that are unresponsive to intralesional steroid therapy ${ }^{48}$. A study also reported on the use of jet injectors for delivering alprostadil for erectile dysfunction in humans ${ }^{49}$, although this study reported that jets produced more pain and bruising and were less effective than needles. Among protein drugs, jet injections have been used to deliver erythropoietin $^{13}$ and interferon ${ }^{14}$ in humans. Other macromolecules, such as low-molecularweight heparin ${ }^{50}$ for treatment of deep vein thrombosis and botulinum toxin ${ }^{51}$ for the treatment of plantar hyperhidrosis, have also been delivered by jet injections in humans.

\section{Emerging applications}

More recently, jet injections have been actively sought for the delivery of DNAbased therapies for various applications ${ }^{52}$. Direct jet injection of naked DNA into tumours in mice has led to tumour regression ${ }^{53,54}$. Intratumoural injection of volumes as low as $1.5-10 \mu \mathrm{l}$ containing

\section{Box 2 | Fluid dynamics of jet formation and penetration}

Impingement of the piston on the drug reservoir in the nozzle of a jet injector increases its pressure from the baseline level to a peak level of $\sim 4,000 \mathrm{psi}$ in less than $0.5 \mathrm{~ms}$. This high pressure forces the liquid through the nozzle at velocities exceeding $100 \mathrm{~m} \mathrm{~s}^{-1}$. The jets used in commercial injectors are typically turbulent with Reynolds numbers (a product of jet diameter, velocity and density divided by viscosity) in the range of tens of thousands. Pressure in the nozzle decreases gradually during skin penetration and steps down suddenly when the entire drug is ejected. The duration of injection is proportional to its volume but the overall jet properties (pressure in the chamber and average jet velocity) are not affected by the amount of liquid loaded in the nozzle. The diameter of the jet is comparable to the nozzle diameter.

The structure of a turbulent jet consists of two significant regions: the initial region within which the fluid travels at the exit velocity; and the main region in which the velocity decreases due to jet expansion ${ }^{77-79}$. The impact of the jet on the skin initiates hole formation in the skin and the velocity of the jet continues to decrease as it travels through the hole. Backflow of the jet is observed during hole formation if the volumetric rate of hole formation in the skin is smaller than the volumetric flow rate of the jet liquid into the skin. Backflow from the hole further slows down the jet entering the hole and decreases the capacity of the jet to penetrate deep into the skin. Beyond a certain depth, the velocity of the jet arriving at the progressive end of the hole drops below the value necessary for hole formation. The hole depth is essentially established at this time. Stagnation pressure of further incoming jet at the end of the hole disperses fluid in a near-spherical manner around the hole.

naked DNA has been used for this purpose ${ }^{54}$. Jet injections have also been used to deliver DNA encapsulated in synthetic or viral vectors. For example, delivery of recombinant adeno-associated virus by jet injection into subcutaneous tumours in mice has been shown to be effective against tumours ${ }^{55}$. Numerous studies have reported the use of jet injection for DNA vaccination in animals ${ }^{56-58}$. The use of jet injectors for DNA vaccination is particularly motivated by the immune competency of skin, especially owing to the presence of Langerhans cells. Jet injections have also been used in combination with other methods such as electroporation for gene delivery ${ }^{59}$. Jetmediated gene delivery is also attractive for the treatment of disorders of keratinocytes, the primary cell type in the epidermis ${ }^{60}$.

\section{Mechanistic considerations}

Physical mechanisms of jet penetration into skin. Although jet injections have been used for the past 60 years ${ }^{61}$ and continue to be researched for new applications, studies on their mechanisms are emerging only now ${ }^{62-65}$. Skin is a non-homogenous, anisotropic material containing various structures of different sizes including the epidermis, which is responsible for skin's barrier function, and the dermis, which is largely responsible for skin's mechanical properties. Accordingly, detailed characterization of events during jet injection is a difficult task. Nevertheless, recent studies have provided significant insight into the mechanisms of jet injections ${ }^{62,64,66}$. Studies have also shed light on the complex fluid dynamics in the injector and in the skin (BOX 2). Mechanistic studies discussed here have been largely performed in vitro using excised skin models. Although it has been suggested that in vitro skin is a reasonable model for in vivo skin ${ }^{62}$, complete evaluation of the relevance of in vitro experimental models needs further consideration.

It is now clear that the impinging jet punctures the skin through erosion and/or fracture mechanisms leading to a clearly identifiable hole ${ }^{66}$ (FIG. 2). Directly visualizing the jet-induced hole is difficult because it is both small and tortuous, as a result of the jet's passage through the non-homogenous layers of the skin; in addition the elastic properties of the skin tend to cause the hole to close on cessation of the jet. Nonetheless, recent studies have made significant progress in measuring the depth ${ }^{66}$ and cross-sectional shape ${ }^{65}$ of the hole. The dimensions of the jet-induced hole are established very early during the injection (a few hundred microseconds after jet impingement on skin) and the terminus of the hole provides a stagnation point for the impinging jet to disperse the rest of the liquid in a near-spherical pattern. Depending on the type of injection (intradermal, subcutaneous or intramuscular) the fluid is deposited primarily in dermis, subcutaneous tissue or muscle. The dermis consists primarily of a matrix of collagen fibres ${ }^{67}$ and deposition of liquid by the jet increases the total volume of the skin, causing it to bulge.

Important jet and skin parameters. Jet-related processes are known to depend on several jet parameters including nozzle diameter, velocity and stand-off distance (the distance between the nozzle and the surface ${ }^{68-71}$. The quality of jet injection also depends on 
a

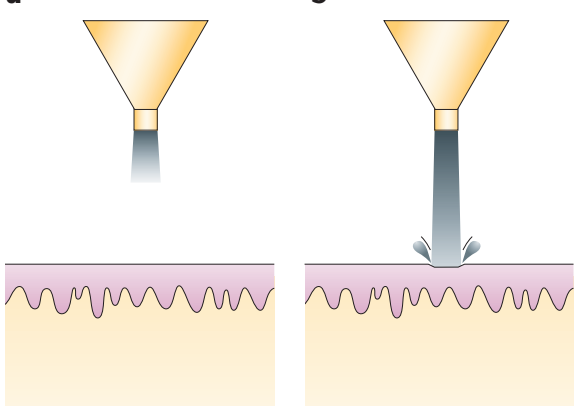

c

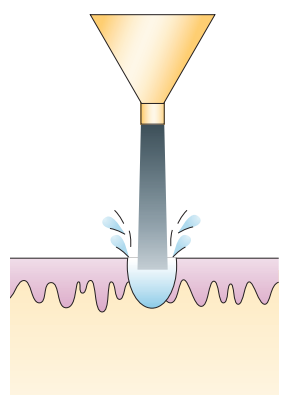

d

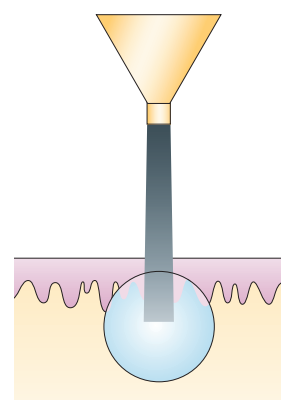

Figure 2 | Schematic depiction of the jet injection process. a | Impact of a piston on a liquid reservoir in the nozzle increases the pressure, which shoots the jet out of the nozzle at high velocity (velocity $>100 \mathrm{~m} \mathrm{~s}^{-1}$ ). $\mathbf{b}$ | Impact of the jet on the skin surface initiates formation of a hole in the skin through erosion, fracture or other skin failure modes. $\mathbf{c}$ |Continued impingement of the jet increases the depth of the hole in the skin. If the volumetric rate of hole formation is less than the volumetric rate of jet impinging the skin, then some of the liquid splashes back towards the injector. $\mathbf{d} \mid$ As the hole in the skin becomes deeper, the liquid that has accumulated in the hole slows down the incoming jet, and the progression of the hole in the skin is stopped. The dimensions of the hole are established very early in the process (a few tens of microseconds) from the time of impact. Stagnation of the jet at the end of the hole disperses the liquid into the skin in a near-spherical shape.

these parameters. Penetration depths of jets into human and animal skin under a few selected jet conditions have long been reported in the literature ${ }^{72,73}$; however, a systematic evaluation of jet penetration under a wide variety of conditions has only recently been attempted ${ }^{64}$.

Completeness of injection (percentage of ejected liquid that is deposited in the tissue) depends primarily on jet velocity and nozzle diameter. Often, a threshold velocity is required to observe detectable penetration into human skin, the value of which depends on nozzle diameter and skin properties. Beyond this threshold, the degree of completeness increases and approaches $100 \%$ at velocities typically used in commercial injectors. For example, at a nozzle diameter of $152 \mu \mathrm{m}$, greater than $90 \%$ delivery was observed in human skin in vitro at a velocity of around $150 \mathrm{~m} \mathrm{~s}^{-1}$ (REF. 62). At a constant velocity (140-160 $\mathrm{m} \mathrm{s}^{-1}$ ), near complete delivery (>90\%) was observed for nozzle diameters in the range of $152-229 \mu \mathrm{m}^{64}$. Slightly reduced completeness $(\sim 80 \%)$ was observed as the nozzle diameter increased or decreased beyond this range $^{62}$. Penetration depth of jets has also been studied over a range of nozzle diameters $(31-559 \mu \mathrm{m})$ and velocities (115-200 $\left.\mathrm{m} \mathrm{s}^{-1}\right)^{64}$. Depending on the jet parameters, the dispersion occurs shallow in the skin $(<<1 \mathrm{~mm})$, deep in the skin $(\sim 2-4 \mathrm{~mm})$ or beyond the dermis. These numbers correspond to low injection volumes $(<100 \mu \mathrm{l})$ and will change if larger volumes are used.

Different combinations of jet exit velocities and nozzle diameters can yield the same jet dispersion pattern. Although neither jet velocity, $\mathrm{U}$, nor nozzle diameter, $\mathrm{D}$, are sufficient to completely characterize the outcome of a jet injection, a combined parameter, exit jet power $(\mathrm{P})$ effectively describes the performance of jet injectors, and is shown in equation 1 (REF. 64):

$P=\frac{1}{8} \pi \rho D^{2} U^{3}$

The existence of a lumped parameter that describes jet penetration brings out the interplay between jet velocity and nozzle diameter. A tenfold increase in $\mathrm{P}$ increases jet penetration into human skin by one millimetre in vitro. Jet delivery also showed a systematic dependence on jet power. Completeness increased linearly with jet power and exceeded $90 \%$ at $\mathrm{P} \sim 30 \mathrm{~W}$ at a constant stand-off distance of $1 \mathrm{~mm}^{64}$ (FIG. 3a). The penetration profile of the jet in the skin also scales with jet power (FIG. 3b).

The skin's mechanical properties also have a strong influence on jet penetration. Several mechanical properties of skin, including Young's modulus (a measure of the stiffness of the skin), critical stress for failure (stress required to rupture skin), fracture toughness and hardness, might have important roles in determining jet penetration. The dependence of penetration and completeness of injection on skin's Young's modulus has been experimentally measured ${ }^{66}$. Penetration depth and completeness decreased with increasing Young's modulus ${ }^{66}$. A threefold increase in skin's Young's modulus decreased the completeness of injection from near $100 \%$ to a mere $10 \%{ }^{66}$.
Drug stability. Drugs are exposed to high shear stresses during injection. Although this is not an issue for low-molecular-weight drugs, it can adversely affect the structural integrity of proteins, vaccines and DNA. The velocities in the nozzle are substantially higher than those in a needle during conventional injections. Furthermore, the diameter of the nozzle is significantly smaller than that of a conventional needle. Collectively, these factors indicate that the shear stresses experienced by drugs inside a jet nozzle are significantly higher than those in a needle. However, the drugs experience high shear in jet injectors for a much shorter period than they do in a needle. Detailed studies are therefore necessary to characterize shearinduced damage in the jet injector. A large body of literature on successful delivery of proteins and DNA, however, suggests that drug disintegration in jet injectors is not an overwhelming concern.

\section{Future prospects}

Needle-free liquid jet injectors have had a significant impact on drug and vaccine delivery. They hold a prominent place in history as an important component of mass immunization programmes and as the first large-scale needle-free method for macromolecule delivery. Jet injectors (DCJIs) provide an attractive alternative to injections. For many needle-phobic patients, they reduce anxiety and offer a preferred mode of delivery. They also eliminate issues related to needles such as accidental punctures and sharps disposal. Compared with other needle-free approaches such as patches, sprays and pills, jet injectors offer an advantage that they can operate with existing formulations designed for needle-based injections. This brings significant cost savings through reduced times of development and clinical trials. However, needle-free liquid jet injectors, despite more than 50 years of clinical use, have not reached their full potential. Several factors contribute to this: occasional pain, discomfort and local reactions ${ }^{61,74}$, inconvenience of use compared with injections and cost.

Limitations of current jet injectors should provide guidelines for future research and development. Fundamental in vitro studies have shown significant variability in jet penetration depending on skin properties ${ }^{62}$. The largest source of variability is likely to be the mechanical properties of the skin, which has a significant role in the outcome of jet injections. Strategies are required to counteract this variability and obtain consistent injections. The development of such 

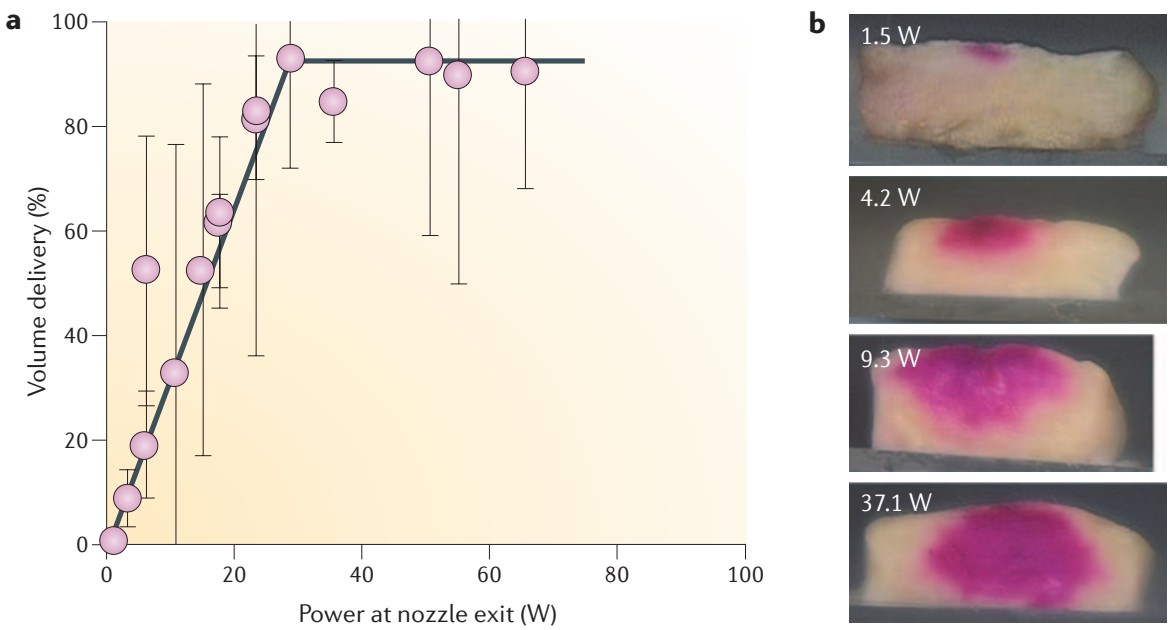

Figure 3 | Dependence of jet penetration on jet parameters. a| Dependence of the volume of jet penetration into human skin on power (see REF. 64 for methods). The percentage of ejected liquid $(80 \mu \mathrm{l})$ that enters the skin increases with increasing power and plateaus at $\sim 30 \mathrm{~W}$. $\mathbf{b}$ | The penetration depth into the skin also depends on jet power. At very low powers (1.5 W) the jet shows extremely shallow penetration (few tens of $\mu \mathrm{m}$ ). As the power increases the jet exhibits a hemispherical $(9.3 \mathrm{~W})$ and spherical dispersion pattern $(37.1 \mathrm{~W})$ in the skin. The actual penetration depth and profile will depend on other parameters, such as ejection volume and stand-off distance, which were held constant in this experiment. Reproduced, with permission, from REF. 64 (c) (2004) Elsevier.

strategies requires a better understanding of the fundamental mechanisms of jet injections. Particular emphasis should be placed on understanding which mechanical properties of the skin affect jet injections. A predictive model describing this phenomenon will also prove valuable.

The occasional pain and bleeding associated with jet injectors also needs to be addressed. The origin of occasional pain is not clear. It is possible that occasional pain and bleeding are associated with variability in a jet's penetration depth. Deep penetration of jets could result in interactions with nerves and blood vessels in the dermal and sub-dermal layer. By restricting jet penetration to the superficial layers of skin, direct interactions with nerves or blood vessels might be potentially minimized. It is also possible that variability in pain is associated with lateral density of nerves and blood vessels. In that case, the use of smaller nozzle diameters could prove helpful.

Currently, there are few differences among the jet parameters used in various commercial jet injectors. The majority use nozzle diameters in the range of 150-300 $\mu \mathrm{m}$ and velocities in the range of 100-200 m $\mathrm{s}^{-1}$. More studies are necessary to explore jet performance outside this range. Particular attention needs to be focused on using smaller nozzle diameters. Reports indicate that patients prefer smaller nozzles, possibly because they are associated with reduced adverse effects ${ }^{10}$; however, mechanistic studies show compromised completeness with smaller nozzles ${ }^{64}$. Efforts should therefore focus on achieving complete delivery using smaller-diameter nozzles. Simultaneous reduction of injection volume is also likely to reduce the occurrence of deep penetration. Low-volume injections are especially attractive for immunization because they naturally target vaccines to the superficial skin layers, which have a high concentration of immune cells ${ }^{75}$. Furthermore, use of low-volume injections also helps in conserving vaccines, a factor that could become important during vaccine shortage. Several additional fundamental questions need to be addressed through further research. For example, the effects of jets on skin at a cellular level are not known. This question is especially relevant in light of the proposed applications of jet injections in genetic immunization.

The design of jet injectors has evolved significantly during the past decade (BOX 1). The introduction of clear, plastic nozzles in DCJIs and fully disposable, pre-loaded devices are perhaps the most notable developments. However, further improvements in jet injector designs are essential to address some of the limitations of existing jet injectors. Newer devices that offer temporal control over injection parameters such as pressure and velocity and produce consistent results despite person to person variation in skin properties are likely to improve the overall quality of injections.
Samir Mitragotri is at the Department of Chemical Engineering, University of California, Santa Barbara, California 93106, USA. e-mail: samir@engineering.ucsb.edu doi: $10.1038 / \mathrm{nrd} 2076$ Published online 23 June 2006

Nir, Y., Paz, A., Sabo, E. \& Potasman, I. Fear of injections in young adults: prevalence and associations. Am. J. Trop. Med. Hyg. 68, 341-344 (2003).

2. Rosenstock, L. CDC's National Institute for Occupational Safety and Health, Committee on Education and the Workforce, Subcommittee on Workforce Protections, US House of Representatives. p. Needlestick Injuries Among Healthcare Workers [online], < http://www.cdc.gov/niosh/ndletest.html > (2000). Kermode, M. Unsafe injections in low-income country health settings: need for injection safety promotion to prevent the spread of blood-borne viruses. Health Promot. Int. 19, 95-103 (2004).

4. Kane, A. et al. Transmission of hepatitis B, hepatitis C and human immunodeficiency viruses through unsafe injections in the developing world: model-based regional estimates. Bull. World Health Organ. 77 801-807 (1999).

5. Tezel, A., Paliwal, S., Shen, Z. \& Mitragotri, S. Low-frequency ultrasound as a transcutaneous immunization adjuvant. Vaccine 23, 3800-3807 (2005).

6. Mitragotri, S. Immunization without needles. Nature Rev. Immunol. 5, 905-916 (2005).

McAllister, D. V. et al. Microfabricated needles for transdermal delivery of macromolecules and nanoparticles: fabrication methods and transport studies. Proc. Natl Acad. Sci. USA 100, 13755-13760 (2003).

8. Choi, W. S. et al. Inhalation delivery of proteins from ethanol suspensions. Proc. Natl Acad. Sci. USA 98 11103-11117 (2001)

9. Langer, R., Where a pill won't reach. Sci. Am. 288, 50-57 (2003)

10. Bremseth, D. L. \& Pass, F. Delivery of insulin by jet injection: recent observations. Diabetes Technol. Ther. 3, 225-232 (2001)

11. Bareille, P. et al. Growth hormone treatment without a needle using the Preci-Jet 50 transjector. Arch. Dis. Child. 76, 65-67 (1997).

12. Dorr, H. G. et al. Are needle-free injections a useful alternative for growth hormone therapy in children? Safety and pharmacokinetics of growth hormone delivered by a new needle-free injection device compared to a fine gauge needle. J. Pediatr. Endocrinol. Metab. 16, 383-392 (2003).

13. Suzuki, T., Takahashi, I. \& Takada, G. Daily subcutaneous erythropoietin by jet injection in pediatric dialysis patients. Nephron 69, 347 (1995).

14. Brodell, R. T. \& Bredle, D. L. The treatment of palmar and plantar warts using natural $\alpha$-interferon and a needleless injector. Dermatol. Surg. 21, 213-218 (1995).

15. Cooper, J. A., Bromley, L. M., Baranowski, A. P. \& Barker, S. G. Evaluation of a needle-free injection system for local anaesthesia prior to venous cannulation. Anaesthesia 55, 247-250 (2000).

16. Florentine, B. D. et al. Local anesthesia for fine-needle aspiration biopsy of palpable breast masses: the effectiveness of a jet injection system. Diagn. Cytopathol. 17, 472-476 (1997).

17. Domino, E. F. et al. A new route, jet injection for anesthetic induction in children. IV. Midazolam plasma levels. Int. J. Clin. Pharmacol. Ther. 36, 458-462 (1998).

18. Domino, E. F. et al. A new route, jet injection for anesthetic induction in children - III. Ketamine pharmacokinetic studies. Int. J. Clin. Pharmacol. Ther 35, 527-530 (1997)

19. Weniger, B. G. Needle-Free Jet Injection Bibliography, Device \& Manufacturer Roster, and Patent List. www. cdc.gov/nip/dev/JetinjeBib.pdf (2004).

20. Weniger, B. G. Jet Injection of Vaccines: Overview and Challenges for Mass Vaccination with Jet Injections (JIs). in Innovative Administration Systems for Vaccines. 2003. Rockville, Maryland: http://www.hhs.gov/nvpo/ meetings/dec2003/Contents/ThursdayPM/Weniger.pdf.

21. Foege, W. H., Millar, J. D. \& Henderson, D. A. Smallpox eradication in West and Central Africa. Bull. World Health Organ. 52, 209-222 (1975).

22. Williams, J. et al. Hepatitis A vaccine administration: comparison between jet-injector and needle injection. Vaccine 18, 1939-1943 (2000). 
23. Canter, J. et al. An outbreak of hepatitis-B associated with jet injections in a weight-reduction clinic. Arch. Intern. Med. 150, 1923-1927 (1990).

24. Weniger, B. G. New High-speed Jet Injectors for Mass Vaccination: Pros and Cons of Disposable-cartridge Jet Injectors (DCJIs) versus Multi-use-nozzle Jet Injectors (MUNJIs), in Global Vaccine Research Forum. 2004, WHO Initiative for Vaccine Research: Montreux, Switzerland.

25. Weintraub, A. M. \& Ponce de Leon, M. P. Potential for cross-contamination from use of a needleless injector. Am. J. Infect. Control 26, 442-445 (1998).

26. Dimache, G. et al. A clinical, epidemiological and laboratory study on avoiding the risk of transmitting viral hepatitis during vaccinations with the Dermojet protected by an anticontaminant disposable device. Vaccine 15, 1010-1013 (1997).

27. Jackson, L. A. et al. Safety and immunogenicity of varying dosages of trivalent inactivated influenza vaccine administered by needle-free jet injectors. Vaccine 19, 4703-4709 (2001)

28. Sarno, M. J. et al. Clinical immunogenicity of measles, mumps and rubella vaccine delivered by the Injex jet injector: comparison with standard syringe injection. Pediatr. Infect. Dis. J. 19, 839-842 (2000).

29. Mathei, C., Van Damme, P. \& Meheus, A. Hepatitis B vaccine administration: comparison between jet-gun and syringe and needle. Vaccine 15, 402-444 (1997)

30. Weller, C. $\&$ Linder, M. Jet injection of insulin vs the syringe-and-needle method. JAMA 195, 844-847 (1966).

31. Katoulis, E. C. et al. Efficacy of a new needleless insulin delivery system monitoring of blood glucose fluctuations and free insulin levels. Int. J. Artif. Organs 12, 333-338 (1989).

32. Lindmayer, I. et al. Development of new jet injector for insulin therapy. Diabetes Care 9, 294-297 (1986)

33. Kerum, G., Profozic, V., Granic, M. \& Skrabalo, Z. Blood glucose and free insulin levels after the administration of insulin by conventional syringe or jet injector in insulin treated type 2 diabetics. Horm Metab. Res. 19, 422-425 (1987).

34. Pehling, G. B. \& Gerich, J. E. Comparison of plasma insulin profiles after subcutaneous administration of insulin by jet spray and conventional needle injection in patients with insulin-dependent diabetes mellitus. Mayo Clin. Proc. 59, 751-754 (1984).

35. Resman, Z., Metelko, Z. \& Skrabalo, Z. The application of insulin using the jet injector DG-77. Acta Diabetol. Lat. 22, 119-125 (1985).

36. Houtzagers, C. M. et al. The Medi-Jector II: efficacy and acceptability in insulin-dependent diabetic patients with and without needle phobia. Diabet. Med. 5, 135-138 (1988)

37. Schneider, U., Birnbacher, R. \& Schober, E. Painfulness of needle and jet injection in children with diabetes mellitus. Eur. J. Pediatr. 153, 409-410 (1994).

38. Rayman, G., Walker, R. \& Day, J. L. Patient experience with a jet injector. Diabet. Med. 6, 274-276 (1989).

39 Theintz, G. E \& Sizonenko, P. C. Risks of jet injection of insulin in children. Eur. J. Pediatr. 150, 554-556 (1991).

40. Verhagen, A., Ebels, J. T., Dogterom, A. A. \& Jonkman, J. H. Pharmacokinetics and pharmacodynamics of a single dose of recombinant human growth hormone after subcutaneous administration by jet-injection: comparison with conventional needleinjection. Eur. J. Clin. Pharmacol. 49, 69-72 (1995)

41. Agerso, H. et al. Pharmacokinetics and pharmacodynamics of a new formulation of recombinant human growth hormone administered by ZomaJet 2 Vision, a new needle-free device, compared to subcutaneous administration using a conventional syringe. J. Clin. Pharmacol. 42, 1262-1268 (2002).
42. Verrips, G. H. et al. Psychological responses to the needle-free Medi-Jector or the multidose Disetronic injection pen in human growth hormone therapy. Acta Paediatr. 87, 154-158 (1998).

43. Zsigmond, E. K., Darby, P., Koenig, H. M. \& Goll, E. F Painless intravenous catheterization by intradermal jet injection of lidocaine: a randomized trial. J. Clin. Anesth. 11, 87-94 (1999).

44. Munshi, A. K., Hegde, A. \& Bashir, N. Clinical evaluation of the efficacy of anesthesia and patient preference using the needle-less jet syringe in pediatric dental practice. J. Clin. Pediatr. Dent. 25 131-136 (2001).

45. Jimenez, N. et al. A comparison of a needle-free injection system for local anesthesia versus EMLA for intravenous catheter insertion in the pediatric patient Anesth. Analg. 102, 411-414 (2006).

46. Greenberg, R. S., Maxwell, L. G., Zahurak, M. \& Yaster, M. Preanesthetic medication of children with midazolam using the Biojector jet injector. Anesthesiology 83, 264-269 (1995).

47. Callen, J. P. Intralesional corticosteroids. J. Am. Acad. Dermatol. 4, 149-151 (1981).

48. Saray, Y. \& Gulec, A. T. Treatment of keloids and hypertrophic scars with dermojet injections of bleomycin: a preliminary study. Int. J. Dermatol. 44 777-784 (2005)

49. Harding, L. M. et al. Comparison of a needle-free highpressure injection system with needle-tipped injection of intracavernosal alprostadil for erectile dysfunction. Int. J. Impot. Res. 14, 498-501 (2002).

50. Hollingsworth, S. J. et al. Delivery of low molecular weight heparin for prophylaxis against deep vein thrombosis using a novel, needle-less injection device (J-Tip). Ann. R. Coll. Surg. Engl. 82, 428-431 (2000).

51. Vadoud-Seyedi, J. Treatment of plantar hyperhidrosis with botulinum toxin type A. Int. J. Dermatol. $\mathbf{4 3}$, 969-971 (2004).

52. Furth, P. A., Shamay, A. \& Hennighausen, L. Gene transfer into mammalian cells by jet injection. Hybridoma 14, 149-152 (1995).

53. Walther, W. et al. Nonviral in vivo gene delivery into tumors using a novel low volume jet-injection technology. Gene Ther. 8, 173-180 (2001)

54. Walther, W. et al. Intratumoral low-volume jet-injection for efficient nonviral gene transfer. Mol. Biotechnol. 21, 105-115 (2002)

55. Janouskova, O. et al. Delivery of recombinant adenoassociated virus by jet injection. Int. J. Mol. Med. 12, 687-691 (2003).

56. Imoto, J. \& Konishi, E. Needle-free jet injection of mixture of Japanese encephalitis DNA and protein vaccines: a strategy to effectively enhance immunogenicity of the DNA vaccine in a murine model. Viral Immunol. 18, 205-212 (2005).

57. Cui, Z, Baizer, L. \& Mumper, R. J. Intraderma immunization with novel plasmid DNA-coated nanoparticles via a needle-free injection device. J. Biotechnol. 102, 105-115 (2003).

58. Ren, S et al. Low-volume jet injection for intradermal immunization in rabbits. BMC Biotechnol. 2, 10 (2002)

59. Horiki, M. et al. Needleless in vivo gene transfer into muscles by jet injection in combination with electroporation. J. Gene Med. 6, 1134-1138 (2004).

60 Sawamura, D. et al. In vivo gene introduction into keratinocytes using jet injection. Gene Ther. $\mathbf{6}$, 1785-1787 (1999).

61. Hingson, R. A., Davis, H. S. \& Rosen, M. The historical development of jet injection and envisioned uses in mass immunization and mass therapy based upon 2 decade's experience. Mil. Med. 128, 516-524 (1963).

62. Schramm, J. R. \& Mitragotri, S. Transdermal drug delivery by jet injectors: energetics of jet formation and penetration. Pharm. Res. 19, 1673-1679 (2002).
63 Schramm-Baxter, J. R , Katrencik, J \& Mitragotri, S Jet injection into polyacrylamide gels: investigation of jet injection mechanics. J. Biomech. 37, 1181-1188 (2004).

64. Schramm-Baxter, J. R. \& Mitragotri, S. Needle-free jet injections: dependence of jet penetration and dispersion in the skin on jet power. J. Control. Release 97, 527-535 (2004)

65. Shergold, O. A., Fleck, N. A. \& King, T. S. The penetration of a soft solid by a liquid jet, with application to the administration of a needle-free injection. J. Biomech. 5 Nov 2005 [epub ahead of print].

66. Baxter, J. \& Mitragotri, S. Jet-induced skin puncture and its impact on needle-free jet injections: experimental studies and a predictive model. J. Control. Release 106, 361-373 (2005)

67. Montagna, W., Bentley, J. P. \& Dobson, R. L. in Advances in Biology of Skin Vol. X (eds Montagna, W. Bentley, J. P. \& Dobson, R. L.) 302 (Appleton-CenturyCrofts, New York, 1970).

68. Yanaida, K. in Proceedings of the Second Internationa Symposium on Jet Cutting Technology A2-19 (BHRA Fluid Engineering, Cambridge, 1974).

69. Yanaida, K. in Fourth International Symposium on Jet Cutting Technology A3-39 (BHRA Fluid Engineering, Canterbury, 1978).

70. Yanaida, K. \& Ohashi, A. in Fifth International Symposium on Jet Cutting Technology 34-44 (BHRA Fluid Engineering, Hanover, 1980).

71. Rajaratnam, N., Steffler, P. M., Rizvi, S. A. H. \& Smy, P. R. An experimental study of very high velocity circular water jets in air. J. Hydraulic Res. 32, 461-470 (1994)

72. Figge, F. H. J. \& Barnett, D. J. Anatomic evaluation of a jet injection instrument designed to minimize pain and inconvenience of parenteral therapy. The Am. Practitioner 3, 197-206 (1948)

73. Bennett, C. R., Mundell, R. D. \& Monheim, L. M. Studies on tissue penetration characteristics produced by jet injection. J. Am. Dental Assoc. 83, 625-629 (1971).

74. Schneider, U., Birnbacher, R. \& Schober, E. Painfulness of needle and jet injection in children with diabetes mellitus. Eur. J. Pediatr. 153, 409-410 (1994)

75. Kupper, T. S. \& Fuhlbrigge, R. C. Immune surveillance in the skin: mechanisms and clinical consequences. Nature Rev. Immunol. 4, 211-222 (2004)

76. Baizer, L., Lacey, C., Hayes, J. \& D'Antonio, L. Needlefree injectors: new technologies. 2001, The Drug Delivery Company Report. Report 2001/02: 51-54

77. Abramovich, G. N. The Theory of Turbulent Jets (MIT Press, Cambridge, 1963).

78. Albertson, M. L., Dai, Y. B., Jensen, R. A. \& Rouse, H. Diffusion of submerged jets. Trans. Am. Soc. Civil Eng. 115, 639-664 (1950).

79. Rajaratnam, N. in Developments in Water Science Vol. 5 (ed. Chow, V. T.) 231-240 (Elsevier, Amsterdam, 1976).

\section{Acknowledgements}

The author acknowledges the scholastic contributions of $\mathrm{J}$. Baxter via original research and valuable discussions. The author also thanks J. Champion, K. Whitehead and A. Arora for help with editing the manuscript. The author apologizes to investigators whose work could not be cited in the article due to reasons of space.

\section{Competing interests statement}

The author declares competing financial interests: see Web version for details.

FURTHER INFORMATION

Centers for Disease Control and Prevention:

http://www.cdc.gov/

World Health Organization: http://www.who.int/en/

Access to this interactive links box is free online.

\section{ERRATUM}

\section{Ranolazine}

Jonathan Abrams, Carole A. Jones and Peter Kirkpatrick

Nature Reviews Drug Discovery 5, 453-454 (2006)

On page 453, information summarizing the efficacy of ranolazine in the ERICA trial was reported incorrectly. The correct information is:

Statistically significant decreases in angina attack frequency and nitroglycerin use were observed in the ranolazine group compared with the placebo group (mean number of attacks/week $=3.3$ and mean number of doses of nitroglycerin/week = 2.7 compared with 4.3 attacks/week and 3.6 doses of nitroglycerin/week, respectively) ${ }^{6}$. 